\title{
Responsiveness and Habituation of Soluble ICAM-1 to Acute Psychosocial Stress in Men: Determinants and Effect of Stress-Hemoconcentration
}

\author{
R. VON KÄNEL ${ }^{1}$, D. PRECKEL ${ }^{2}$, B. M. KUDIELKA ${ }^{3}$, J. E. FISCHER ${ }^{4}$ \\ ${ }^{1}$ Department of General Internal Medicine, University Hospital Bern, Switzerland, ${ }^{2}$ Institute for \\ Behavioral Sciences, Federal Institute of Technology, Zürich, Switzerland, ${ }^{3}$ Department of \\ Theoretical and Clinical Psychobiology, University of Trier, Germany, ${ }^{4}$ Department of Public \\ Health, Social and Preventive Medicine, Mannheim Medical Faculty, University of Heidelberg, \\ Mannheim, Germany
}

Received May 24, 2006

Accepted August 23, 2006

On-line available December 19, 2006

\begin{abstract}
Summary
We studied the psychophysiology of soluble intercellular adhesion molecule-1 (sICAM-1) in 25 apparently healthy middle-aged men who underwent an acute psychosocial stressor three times with one week apart. Measures of the biological stress response were obtained at week one and three. The magnitude of the sICAM-1 stress response showed no habituation between individual visits. At week one, cognitive stress appraisal independently predicted integrated sICAM-1 area under the curve (AUC) between rest, immediately post-stress, and 45 min and 105 min post-stress $\left(\beta=0.67, p=0.012, \Delta R^{2}=0.41\right)$. Diastolic blood pressure AUC $\left(\beta=-0.45, p=0.048, \Delta R^{2}=0.21\right)$ and heart rate AUC $\left(\beta=0.44, \quad p=0.055, \Delta R^{2}=0.21\right)$ were independent predictors of sICAM-1 AUC at week three. Adjustment for hemoconcentration yielded a decrease in sICAM-1 levels from rest to post-stress $(\mathrm{p}<0.001)$. Stress responsiveness of plasma sICAM-1 was predicted by stress perception and hemodynamic reactivity and affected by stresshemoconcentration but unrelated to cortisol reactivity and not readily adapting to repeated stress.
\end{abstract}

Key words

Adhesion molecule • Hypothalamic-pituitary-adrenal axis $\bullet$ Psychological stress

\section{Introduction}

Cellular adhesion molecules (CAM) mediate adherence and subsequent transmigration of circulating leukocytes across the vascular endothelium (Price and Loscalzo 1999) pertinent to vascular inflammation and early atherosclerosis (Davies et al. 1993). A major member of the CAM superfamily is the glycoprotein intercellular adhesion molecule-1 (ICAM-1) that is constitutively expressed on endothelial cells and upregulated by inflammatory cytokines (Wertheimer et al. 1992) to become a firm ligand for lymphocyte functionassociated antigen-1 expressed on activated leukocytes (Marlin and Springer 1987). The soluble form of ICAM-1 
(sICAM-1) reflects ICAM-1 expression on endothelial cells (Leeuwenberg et al. 1992) and, therefore, provides an easy-to-measure clinical marker of vascular inflammation and endothelial activation (Witkowska 2005). Circulating ICAM-1 levels correlated with the extent of atherosclerosis in carotid arteries (Rohde et al. 1998) and predicted recurrent coronary events in patients with chronic coronary artery disease (Haim et al. 2002). In apparently healthy men, sICAM-1 increased the prospective risk of developing first time myocardial infarction (Ridker et al. 1998) and peripheral arterial disease (Pradhan et al. 2002).

Modulation of circulating ICAM-1 levels by the sympathetic nerve and adrenergic function, respectively, is potentially important for cardiovascular diseases (Mills et al. 2002). For instance, the acute onset of myocardial infarction due to physical and mental stress is wellestablished (Mittleman et al. 1993, 1995), and early increase in sICAM-1 in men admitted with acute chest pain predicted greater risk of acute coronary syndromes (O’Malley et al. 2001). Dynamic exercise increased sICAM-1 levels and pretreatment with either $\beta_{1}$ - or $\beta_{2}$ adrenergic blockade mitigated this effect (Rehman et al. 1997). However, sICAM-1 was not responsive to exercise in healthy men when post-exercise sICAM-1 levels were adjusted for changes in plasma volume (Timmons et al. 2006). Acute psychological stress in a natural setting also provoked a significant increase in sICAM-1 levels (Dugue et al. 1999, Heinz et al. 2003) that was maintained after adjustment for hemoconcentration (Dugue et al. 1999). However, infusion of the nonselective $\beta$-adrenergic agonist isoproterenol did not change sICAM-1 levels (Mills et al. 2002), which is compatible with the finding that $\beta_{2}$-adrenergic agonists inhibited expression of ICAM-1 on monocytes stimulated with lipopolysaccharide (LPS) (Kuroki et al. 2004). These data suggest that stress-induced shear stress (Niebauer and Cooke 1996), but not adrenergic activation per se (Mills et al. 2002), results in endothelial shedding of ICAM-1 (Sultan et al. 2004) into the circulation (Rehman et al. 1997) and that interpretation of SICAM-1 levels in cardiovascular stress experiments warrants considering the effect of hemoconcentration (Allen and Patterson 1995).

As opposed to the sympathomedullary system, the role of the hypothalamic-pituitary-adrenal (HPA) axis in regulation of sICAM-1 during stress is largely unknown. In vitro suppression of LPS-stimulated cytokine release from leukocytes by dexamethasone resulted in decreased ICAM-1 expression on endothelial cells (Antonelli et al. 2000). Furthermore, glucocorticoids suppressed sICAM-1 release from cytokine-stimulated cultured human bronchial epithelial cells (Wang et al. 1997) and reduced elevated SICAM-1 levels in patients with rheumatic disease (Macchioni et al. 1994), but increased sICAM-1 in septic shock patients (Leone et al. 2004). In healthy men, cortisol increase during exercise (Timmons et al. 2006) and acute psychological stress (Heinz et al. 2003) were unrelated to sICAM-1 levels.

To further elucidate stress physiology of sICAM-1, we investigated its responsiveness to acute psychosocial stress and recovery in a habituation experiment. The potential of humans to habituate to the same repeated stressor is viewed as an adaptive performance of the organism, protecting it from overshooting biological stress responses (McEwen 1998). Given the inconsistent effects of glucocorticoids and cortisol on sICAM-1, we primarily aimed to investigate stress-induced changes in sICAM-1 in relation to reactivity of the HPA axis and stress-hemoconcentration, and to ascertain whether stress perception would affect sICAM-1 responsiveness. The transactional model of stress (Lazarus and Folkman 1984) asserts that the meaning of a stressor and associated psychophysiological reactions are determined by an interaction of a persons' interpretation of a situation based on perceived coping skills and characteristics of the stressor (Park 1998). We hypothesized that SICAM-1 response changes significantly from rest to post-stress, fully restores during recovery, and habituates (i.e. decreases) across visits. We further hypothesized that SICAM-1 reactivity shows a positive association with greater stress appraisal, and that the time course of sICAM-1 levels during the stress experiment changes with adjustment to the plasma volume shift.

\section{Methods}

\section{Study participants}

The ethics committee of the Federal Institute of Technology, Zurich, Switzerland formally approved the study protocol and all participants provided their written consent. The detailed recruitment procedure yielding 27 apparently healthy men $>35$ years of age of the Swiss Federal Institute of Technology has previously been described (von Känel et al. 2004). Because of occasional assay problems and clotted lines, we report in the present study data from 25 subjects who had complete sICAM-1 measures for all four time points of the stress experiment at week one. Two subjects dropped out of the protocol 
after the first visit due to time restriction such that we report sICAM-1 measures at week three from 23 subjects. To verify that all participants were healthy (i.e. normal immune function), we took their medical history and performed physical examination as well as routine laboratory work-up (Synlab, Augsburg, Germany). Specified exclusion criteria were: any hematological, pulmonary, gastrointestinal, liver, renal, heart, cerebrovascular, or psychiatric disease; any history of a thromboembolic event, any current major or minor infection, any trauma or surgery within the preceding six months, body mass index $\geq 29 \mathrm{~kg} / \mathrm{m}^{2}$, and high-sensitive C-reactive protein $\geq 1 \mathrm{mg} / \mathrm{dl}$. The absence of a major psychiatric disorder was verified by asking the question "have you ever been diagnosed with a psychiatric disorder?" and by self-reported symptom levels of depression and anxiety during the previous week (von Känel et al. 2005). Moreover, all subjects were not medicated and were required not to take any nonsteroidal anti-inflammatory drug for at least ten days before testing and throughout the habituation protocol. All participants had anthropometric data and screening blood pressure (BP) assessed.

After termination of the habituation protocol we recruited a sample of healthy age-matched male controls from the same cohort who were not stressed and who were not different in subject characteristics from the stress group (von Känel et al. 2006). The main purpose of including a control group was to verify that changes in sICAM-1 were indeed elicited by psychosocial stress and not by diurnal variation. In ten controls, blood for measuring sICAM-1 was obtained once at the same time points and processed in the same way as in the stress group.

\section{Stress protocol}

Participants were scheduled three times with an interval of one week. Either two or three subjects were tested in the morning starting at 07:30 h and with an interval of 30 to $45 \mathrm{~min}$ between subjects. They had refrained from smoking and exercising since the previous evening. After arrival at our laboratory, subjects had a 20gauge venous catheter placed in their forearm and received a light, standardized, non-caffeinated breakfast. They remained seated for another $30 \mathrm{~min}$ until given instructions about the stress protocol. We applied the Trier Social Stress Test (TSST) that combines a 3-min preparation phase followed by 5 -min free speech (mock job interview) and 5-min mental arithmetic before an audience (Kirschbaum et al. 1993). Specifically, the "preparation phase" refers to the first phase of the TSST during which subjects are requested to prepare their upcoming talk in front of the jury. After completion of the task, subjects remained seated in a quiet room for another $105 \mathrm{~min}$. After the first visit (week one), subjects were informed that the two subsequent visits (weeks two and three) would be identical. However, we slightly modified the topic of the speech and the initial number for the serial subtraction tasks across visits to avoid effects of learning (Schommer et al. 2003).

\section{Biological stress measures}

Blood samples for sICAM-1 measures were obtained immediately before the preparation phase, immediately post-stress, and after $45 \mathrm{~min}$ and $105 \mathrm{~min}$ of recovery from stress. After discarding the first $2 \mathrm{ml}$, venous blood was collected into polypropylene test tubes containing $3.8 \%$ sodium citrate (Vacutainer, Becton Dickinson, Allschwil, Switzerland) and centrifuged at $3000 \mathrm{xg}$ for $10 \mathrm{~min}$ at room temperature. Obtained plasma was aliquoted into polypropylene tubes and frozen at $-80{ }^{\circ} \mathrm{C}$ until further analysis. Funding allowed assessment of sICAM-1 at week one and week three but not at week two. Plasma levels of sICAM-1 were determined by ELISA according to manufacturer instructions (BioSource International, Inc., Camarillo, CA, USA). Intra- and inter-assay coefficients of variation were $<10 \%$. All post-stress sICAM-1 levels were then recalculated with adjustment for stress-induced plasma volume shift using a previous formula (Dill and Costill 1974). Hematocrit was automatically calculated from the erythrocyte concentration and the impedance-determined mean corpuscular volume of erythrocytes. Hemoglobin concentration was measured by the cyanomethemoglobin method.

Saliva for cortisol measures was assessed immediately before and post-stress, and 15, 30, 45 and $105 \mathrm{~min}$ thereafter. Saliva samples were stored in polypropylene Eppendorf tubes at $-20^{\circ} \mathrm{C}$ and, following thawing, centrifuged at $3000 \mathrm{rpm}$ for $5 \mathrm{~min}$ to obtain clear supernatant fractions. Determination of free salivary cortisol was by Luminescence Immuno Assay (LIA) kit supplied by IBL (Hamburg, Germany) (Westermann et al. 2004).

Systolic BP (SBP) and diastolic BP (DBP) were measured by sphygmomanometry immediately before stress and $45 \mathrm{~min}$ thereafter. During stress, BP values were computed as the highest mean value determined from at least five valid readings by the Vasotrac Model APM205A (Medwave, St. Paul, MN, USA) and adjusted 
to sphygmomanometry readings as previously described (von Känel et al. 2004). Mean heart rate (HR) values immediately pre- and post-stress, and $45 \mathrm{~min}$ and 105 min thereafter were computed from 10 stable and digitally recorded R-R intervals over a period of 30 seconds (400 Hz electrocardiogram lead).

Table 1. Characteristics of 25 healthy men studied (mean \pm SD, range)

\begin{tabular}{|c|c|}
\hline Age [years] & $47 \pm 8(38-59)$ \\
\hline \multicolumn{2}{|l|}{ Level of education } \\
\hline did not finish primary school & 0 \\
\hline graduated from primary school & 2 \\
\hline graduated from secondary school & 6 \\
\hline graduated from high school & 17 \\
\hline \multicolumn{2}{|l|}{ Smoking status } \\
\hline current smoker & 3 \\
\hline quit smoking $>5$ years ago & 2 \\
\hline quit smoking $>10$ years ago & 6 \\
\hline life-long non-smoker & 14 \\
\hline \multicolumn{2}{|l|}{ Physical exercise } \\
\hline exercises regularly $>2 h / w k$ & 11 \\
\hline exercises regularly $1-2 \mathrm{~h} / \mathrm{wk}$ & 3 \\
\hline exercises regularly $<1 \mathrm{~h} / w k$ & 6 \\
\hline no exercise & 5 \\
\hline Alcohol consumption [days/week] & $2.3 \pm 1.7(0-6)$ \\
\hline Body mass index $\left[\mathrm{kg} / \mathrm{m}^{2}\right]$ & $24.6 \pm 2.4(19.6-28.9)$ \\
\hline Waist-to-hip ratio & $0.87 \pm 0.10(0.73-1.00)$ \\
\hline Total serum cholesterol $[\mathrm{mg} / \mathrm{dl}]$ & $203 \pm 31(160-299)$ \\
\hline Low-density lipoprotein cholesterol [mg/dl] & $128 \pm 28(81-200)$ \\
\hline High-density lipoprotein cholesterol [mg/dl] & $51 \pm 10(37-73)$ \\
\hline Serum triglycerides $[\mathrm{mg} / \mathrm{dl}]$ & $120 \pm 46(63-243)$ \\
\hline Systolic blood pressure [mmHg] & $126 \pm 14(107-155)$ \\
\hline Diastolic blood pressure [mmHg] & $82 \pm 8(67-97)$ \\
\hline Glycosylated hemoglobin Alc [\%] & $4.9 \pm 0.5(4.1-6.7)$ \\
\hline
\end{tabular}

Table 2. Hierarchical regression analyses for area under the curves of sICAM-1

\begin{tabular}{llllll}
\hline Variables entered & $\begin{array}{l}\text { Partial } \\
\text { correlations }\end{array}$ & $\begin{array}{l}\text { Standardized } \\
\beta \text {-coefficient }\end{array}$ & $\boldsymbol{t}$ & $\boldsymbol{P}$ & $\boldsymbol{R}^{2}$ change \\
\hline sICAM-1 area under the curve at week one & & & & 0.003 \\
Cortisol AUC & 0.20 & 0.16 & 0.71 & 0.489 & 0.031 \\
Heart rate AUC & 0.26 & 0.23 & 0.95 & 0.361 & 0.000 \\
Diastolic BP AUC & -0.18 & -0.15 & -0.62 & 0.548 & 0.406 \\
Total stress score & 0.65 & 0.67 & 2.95 & 0.012 & 0.010 \\
sICAM-1 area under the curve at week three & -0.01 & & & 0.214 \\
Cortisol AUC & -0.02 & 0.44 & -0.06 & 0.950 & 0.205 \\
Heart rate AUC & 0.46 & -0.45 & 2.07 & 0.055 & 0.001 \\
Diastolic BP AUC & -0.47 & 0.03 & -2.14 & 0.048 & 0.14 \\
Total stress score & 0.03 & & 0.14 & \\
\hline
\end{tabular}

AUC, area under the curve; BP, blood pressure; sICAM-1, soluble intercellular adhesion molecule-1 
Table 3. Items of the stress perception scale

\begin{tabular}{lll}
\hline Item \# & Anticipatory cognitive stress appraisal & Retrospective cognitive stress appraisal \\
\hline 1 & I find the pending situation a challenge & I found the situation challenging \\
2 & I find the pending situation strenuous & I found the situation strenuous \\
3 & I feel in control of the pending situation & I felt in control of the situation \\
4 & I find the pending situation difficult & I felt the situation to be demanding \\
5 & I find the pending situation demanding & I showed a good performance \\
6 & I will show a good performance & It was important to me to master the situation \\
7 & It is important for me to master the pending & \\
8 & situation well & The situation was new to me \\
9 & I find the pending situation threatening & I found the situation threatening \\
\hline
\end{tabular}

\section{Stress perception scale}

We construed a stress scale relating to the TSST following a transactional stress model (Lazarus and Folkman 1984). In brief, this model proposes that people's interpretations of an event, their personal resources, and characteristics of the stressor interact to determine the subjective meaning of the stressor, subject's coping efforts and, importantly, also their psychophysiological reactions (Park 1998).

Individual items of the stress questionnaire are presented in Table 3 (for item presentation, a native English speaking person also fluent in German translated items from the German language into English). Because anticipatory and retrospective cognitive stress appraisal may differently relate to e.g. HPA axis response to stress (Gaab et al. 2005), we applied the same questionnaire twice, once before and once after the subjects had undergone the TSST using appropriate wording. The questionnaire asked for nine items rated on a Likert scale ranging from 1 ("not at all") to 7 ("absolutely"). Items \# 3 and \# 6 were inversely coded. We computed a total measure of transactional stress perception adding up and averaging individual item scores obtained pre- and poststress (range 1 to 7 points) and two subscales of anticipatory and retrospective stress appraisal.

For the anticipatory and retrospective subscale, the total score of items meets the mutual dependence of subjects' perception of and perceived skills to cope with the stressor according to Lazarus' theory. Thus, a higher total score means a higher general stress perception. The total score of items was calculated and used for analyses because it yields a more robust measure of stress perception than do individual items and because it avoids potentially spurious associations due to multiple comparisons with the use of individual items. Psychometric evaluation showed good internal consistency for the total stress score (mean Cronbach's $\alpha$ across all three visits $=0.84$ ) and reasonable consistency for both the anticipatory (mean $\alpha=0.72$ ) and retrospective (mean $\alpha=0.74$ ) stress appraisal scores.

\section{Statistical analyses}

Data were analyzed using SPSS (version 12.0) statistical software package (Chicago, IL, USA) with significance level set at $\mathrm{p} \leq 0.05$; all tests were two-tailed. Data are presented as means \pm S.D. in tables and text and as means \pm S.E.M. in figures. As per the KolmogorovSmirnov test all metric data showed a normal distribution. Bivariate correlation analyses used Pearson estimates.

Repeated measures analysis of variance was used to investigate significant within- and betweensubject effects with the TSST and between visit one (week one) and visit three (week three). Multivariate testing applied Roy's largest Root and accounted for violation of the sphericity assumption applying the Greenhouse-Geiser correction of degrees of freedom. For post hoc analyses, sICAM-1 and plasma volume measures of both visits were aggregated in order to yield more robust individual differences in the stress response of these variables (Kamarck et al. 2000). Fisher's Least Significant Difference was used for post hoc analyses. Data were occasionally missing due to technical problems.

To compute the area under the curve (AUC) of biological stress measures across all time points we used a formula which considers the variable time between measurements and ignores the distance from zero for all 
measurements, thereby emphasizing a variable's integrated change over time (Pruessner et al. 2003). Hierarchical linear regression analysis (enter method) was applied to investigate the predictive value of stress perception and integrated changes in cortisol and hemodynamic reactivity for integrated change over time in sICAM-1 levels. We computed two equations in which cortisol $_{\mathrm{AUC}}, \mathrm{HR}_{\mathrm{AUC}}, \mathrm{DBP}_{\mathrm{AUC}}$, and total stress score (i.e. sum of the anticipatory plus the retrospective stress appraisal score) were regressed on $\mathrm{sICAM}-1_{\mathrm{AUC}}$ in this order at respective visits to identify independent predictors of $\mathrm{sICAM}-1_{\mathrm{AUC}}$ measures. Cortisol $_{\mathrm{AUC}}$ was retained in equations a priori as an indicator of HPA axis activity, whereas $\mathrm{SBP}_{\mathrm{AUC}}$ was not entered as a covariate because it was not significantly associated with sICAM-1 AUC measures in bivariate analyses. In a final step we investigated the relationship between sociodemographic, lifestyle, and medical variables with sICAM- $1_{\text {AUC }}$ of both visits to identify whether these would confound the predictive value of perceived stress and hemodynamic measures for $\mathrm{SICAM}-1_{\mathrm{AUC}}$ measures.

\section{Results}

\section{Subjects' characteristics}

Table 1 presents the demographic and medical data of subjects showing that our middle-aged men were in reasonably good cardiovascular health and had a fairly high socioeconomic status. The Wilson-Framingham index (Wilson et al. 1998) predicted an average risk of $7 \pm 2 \%$ for the development of CAD ten years down the line confirming good health of subjects.

\section{Stress reactivity and habituation effect}

There were significant time-by-stress effects for HR $\quad\left(\mathrm{F}_{1.3,27.5}=56.8, \quad \mathrm{p}<0.001 ; \quad\right.$ Fig. $\left.1 \mathrm{~A}\right), \quad$ DBP $\left(\mathrm{F}_{1.3,20.1}=170.5, \quad \mathrm{p}<0.001 ; \quad\right.$ Fig. $\left.1 \mathrm{C}\right), \quad$ and sICAM-1 $\left(F_{2.555 .3}=10.7, p<0.001\right.$; Fig. 1D). There were, however, no significant time-by-stress-by-visit interactions for $\mathrm{HR}$ $\left(\mathrm{F}_{2.8,25.2}=1.2, \mathrm{p}=0.33\right)$, DBP $\left(\mathrm{F}_{1.4,258.5}=3.3, \mathrm{p}=0.072\right)$; and sICAM-1 $\left(\mathrm{F}_{2.7,21.2}=0.6, \mathrm{p}=0.98\right)$ suggesting that the magnitude of the stress response in these three measures showed no significant habituation from week one to week three. A habituation effect in the magnitude of stress responses (i.e. time-by-stress-by-visit interaction) was found in terms of $\operatorname{SBP}\left(\mathrm{F}_{1.9,30.3}=8.8, \mathrm{p}=0.001\right.$; Fig. 1B) and cortisol levels $\left(\mathrm{F}_{3.1,67.2}=4.1, \mathrm{p}=0.010\right.$; Fig. 1E). Peak responses in SBP $(p<0.001$; Fig. 1B) and in cortisol $30 \mathrm{~min}(\mathrm{p}=0.011)$ and $45 \mathrm{~min}(\mathrm{p}=0.003)$ post-stress (Fig.
1E) were weaker at week three than at week one.

Post hoc analyses aggregating sICAM-1 observations of both weeks (i.e. 25 observations of week one plus 23 observations of week three) showed that sICAM-1 increased between rest and immediately post stress $(p=0.040)$ and that it was lower after $45 \mathrm{~min}$ $(p=0.024)$ and $105 \mathrm{~min}(\mathrm{p}=0.017)$ of recovery than at rest. In addition, there was a significant time-by-visit interaction for sICAM-1 levels $\left(\mathrm{F}_{1,22}=14.1, \mathrm{p}=0.001\right)$; sICAM-1 levels were higher at week three than at week one at all four time points (all $\mathrm{p} \leq 0.004$; Fig. 1D). Control subjects showed no significant change in their sICAM-1 levels across the same four time points $\left(\mathrm{F}_{1.9}\right.$, 717.2 $=1.9, \mathrm{p}=0.18$ ) suggesting that change in sICAM-1 with the stress protocol reflected stress responsiveness of sICAM-1 and not a circadian phenomenon.

Total stress appraisal score did not change between visit one and visit three $(p=0.12)$. However, there was a significant time-by-stress-by-visit interaction (i.e. habituation effect) for perceived stress levels pre- vs. post-stress $\quad\left(\mathrm{F}_{1,22}=18.5, \mathrm{p}<0.001 ; \quad \mathrm{Fig} .1 \mathrm{~F}\right)$. While anticipatory stress appraisal was similar between testing sessions, the magnitude of retrospective stress appraisal diminished from week one to week three $(p<0.001)$. Exploratory analysis of individual items particularly revealed a drop from week one to week three in retrospectively appraised importance of mastering the situation (item \# 7; $5.1 \pm 1.4$ vs. $4.6 \pm 1.8, \mathrm{p}=0.015$ ) and novelty of the situation (item \# $8 ; 5.0 \pm 2.1$ vs. $2.9 \pm 2.4$, $\mathrm{p}=0.006)$.

\section{Predictors of change in soluble ICAM-1}

Bivariate correlation analyses showed that SICAM-1 AUC at week one correlated with total stress perception $(\mathrm{r}=0.56, \mathrm{p}=0.003$; Fig. 2A) but not significantly with cortisol $_{\mathrm{AUC}}, \mathrm{HR}_{\mathrm{AUC}}, \mathrm{SBP}_{\mathrm{AUC}}$ and $\mathrm{DBP}_{\mathrm{AUC}}$ of week one. More detailed analysis showed that anticipatory $(\mathrm{r}=0.50, \mathrm{p}=0.012)$ and retrospective $(\mathrm{r}=0.45$, $\mathrm{p}=0.025$ ) stress perception were both correlated with sICAM-1 $1_{\text {AUC. }}$ At week three, sICAM-1 $1_{\text {AUC }}$ correlated with $\operatorname{HR}_{\mathrm{AUC}}(\mathrm{r}=0.50, \mathrm{p}=0.016$; Fig. $2 \mathrm{~B})$ and with $\mathrm{DBP}_{\mathrm{AUC}}$ $(\mathrm{r}=-0.50, \mathrm{p}=0.021$; Fig. $2 \mathrm{C})$ but not with cortisol $_{\mathrm{AUC}}$, $\mathrm{SBP}_{\mathrm{AUC}}$, and total stress perception.

In linear regression analyses, the total stress score emerged as the only significant predictor of sICAM- $1_{\mathrm{AUC}}$ at week one (Table $2 \mathrm{a}$ ). At week three, $\mathrm{DBP}_{\mathrm{AUC}}$ significantly predicted s-ICAM-1 $1_{\mathrm{AUC}}$ and $\mathrm{HR}_{\mathrm{AUC}}$ reached a trend towards statistical significance as an independent predictor of sICAM-1 (Table 2b). Removing 

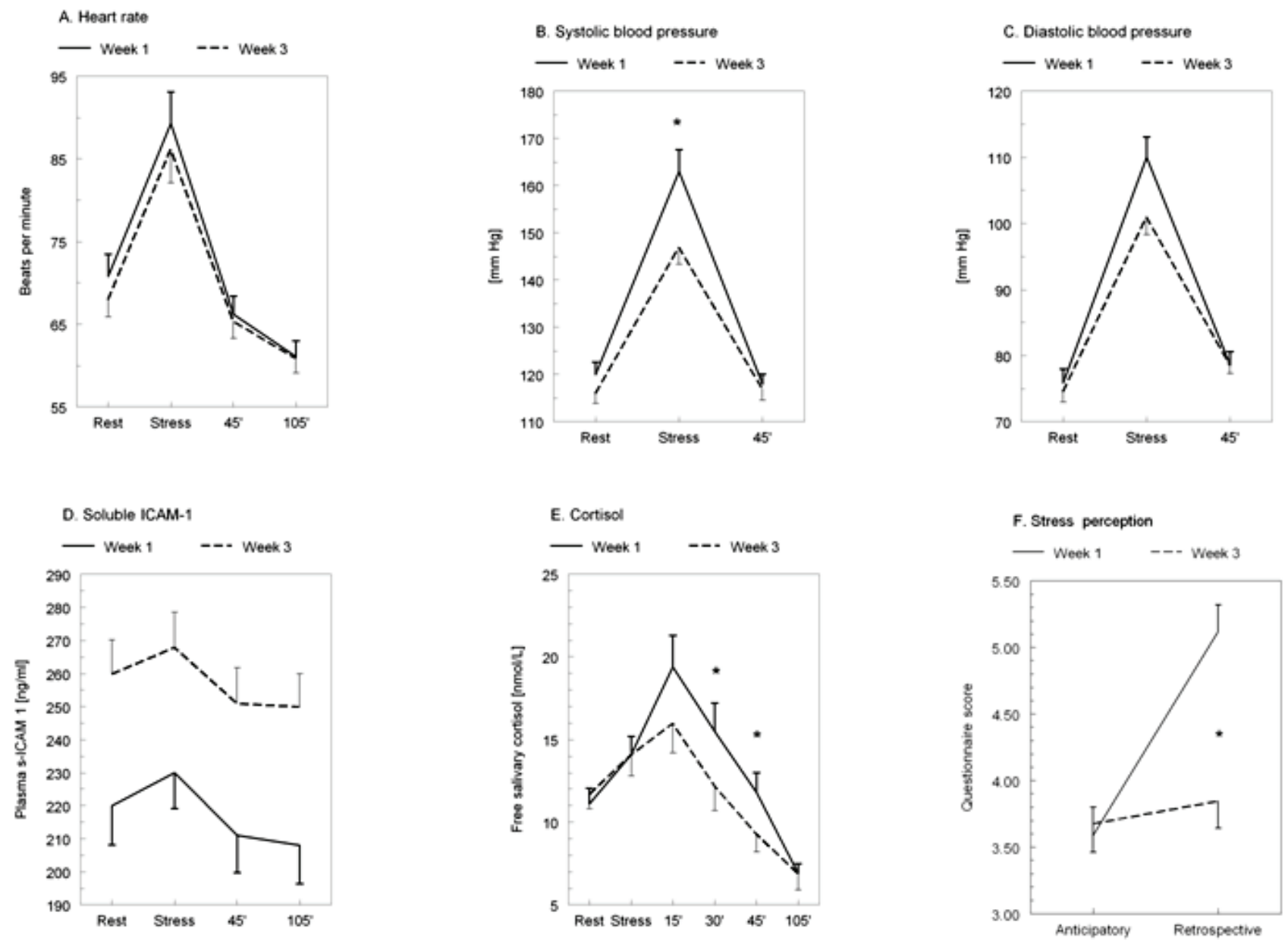

Fig. 1. Change in biological markers and stress perception between sessions. Stress provoked a significant change over time in all variables measured (means \pm SEM). While the magnitude of the stress response did not differ between testing sessions in terms of heart rate $(n=22)$, diastolic blood pressure $(n=17)$, and soluble intercellular adhesion molecule-1 (sICAM-1) $(n=23)$, there emerged significant habituation effects $(*)$ in terms of systolic blood pressure $(n=17)$, cortisol activity $(n=23)$, and appraisal of stress ( $n=23$ ) between testing sessions.

cortisol $_{\mathrm{AUC}}$ from equations did not change the significance of any predictor for $\mathrm{SICAM}-1_{\mathrm{AUC}}$ measures of both visits (data not shown in detail).

There were no significant relationships between either of the sICAM-1 measures and age, education, smoking status, exercise, alcohol, BMI, WHR, total cholesterol, LDL cholesterol, HDL cholesterol, triglycerides, SBP, DPB, HbAlc, and the WilsonFramingham Index of CAD risk (all $p \geq 0.10$ ). We therefore did not control for any of these variables as covariates in regression equations.

\section{Effect of hemoconcentration on SICAM-1}

Figure 3 shows the significant time-by-stress interaction for plasma volume change $\left(\mathrm{F}_{1,19}=8.7\right.$, $\mathrm{p}=0.008$ ) and the significant time-by-stress interaction for sICAM-1 corrected for plasma volume shift $\left(\mathrm{F}_{2.8,53.4}=31.3, \mathrm{p}<0.001\right)$ across time points. There were, however, no significant time-by-stress-by-visit interactions for both plasma volume change $\left(\mathrm{F}_{2.5,47.9}=0.05, \mathrm{p}=0.97\right)$ and sICAM-1 corrected for plasma volume shift $\left(\mathrm{F}_{2.7,51.2}=0.07, \mathrm{p}=0.97\right)$ suggesting that the magnitude of the stress response in these parameters did not undergo habituation between visits.

Post hoc analyses using aggregated measures of plasma volume (23 observations of week one plus 22 observations of week two) and of corrected sICAM-1 (23 observations of week one plus 22 observations of week three) showed a significant decrease of plasma volume and corrected sICAM-1 levels from rest to immediately post-stress ( $p<0.001$ both). The direction of sICAM-1 time course corrected for plasma volume shift mirrored the time course of uncorrected sICAM-1 levels (Fig. 1D). Full recovery of stress-induced changes in plasma volume and in corrected sICAM-1 levels was achieved 45 min post-stress. However, whereas corrected 

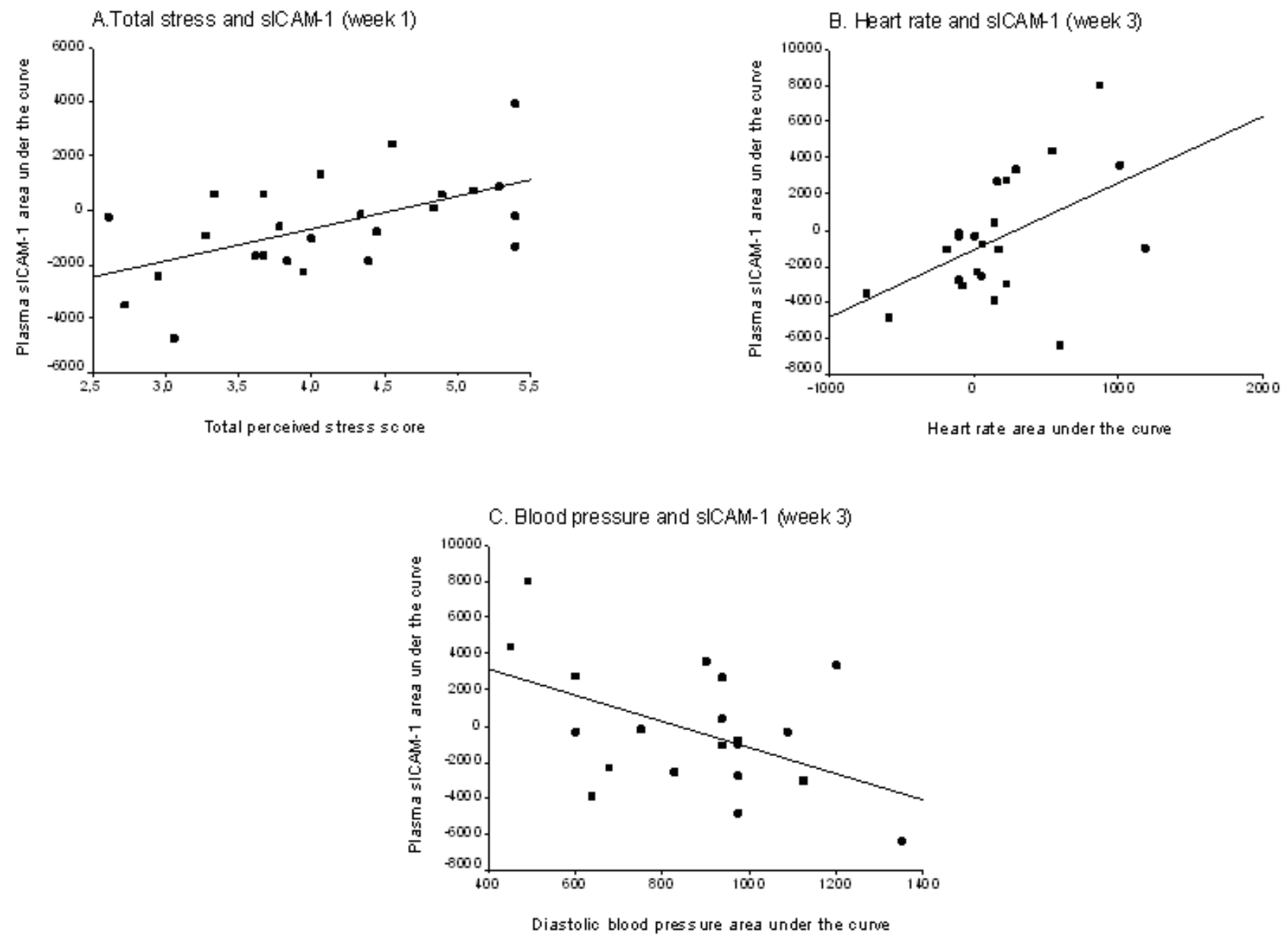

Fig. 2. Association of stress perception and hemodynamics with SICAM-1 change. Panels show significant positive associations with fit line between total stress score and soluble intercellular adhesion molecule- 1 area under the curve (sICAM-1 $1_{\text {AUC }}$ ) at week one (Panel A, $\mathrm{n}=25$ ), between heart rate ${ }_{\text {AUC }}$ and sICAM- $1_{\text {AUC }}$ at week three (Panel $\left.B, n=25\right)$, and between diastolic blood pressure ${ }_{\text {AUC }}$ and sICAM- $1_{\text {AUC }}$ at week three (Panel $\mathrm{C}, \mathrm{n}=23$ ). Auc units are arbitrary.
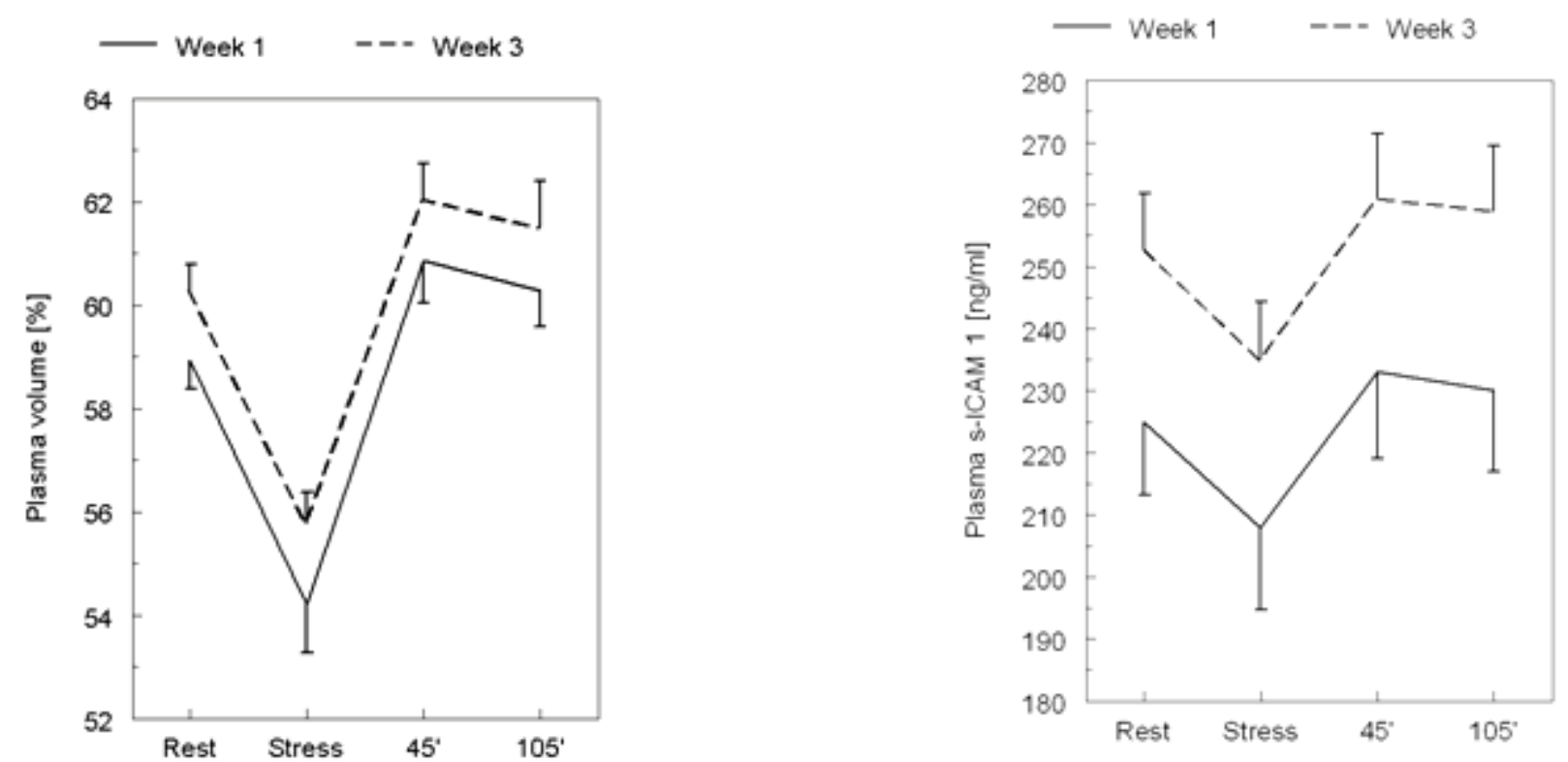

Fig. 3. Stress response of plasma volume and sICAM-1 corrected for plasma volume shift. The figure shows the stress-induced change in levels of plasma volume $(n=20)$ and in soluble intercellular adhesion molecule $(\operatorname{sICAM})-1(n=20)$ corrected for plasma volume shift (means $\pm \mathrm{SEM}$ ). 
sICAM-1 levels were similar 105 min post-stress compared to rest, plasma volume was higher $105 \mathrm{~min}$ after stress than at rest $(\mathrm{p}=0.025)$.

Area under the curve measures of cortisol, HR, SBP, DBP and total stress scores showed no significant correlations with $\mathrm{sICAM}-1_{\mathrm{AUC}}$ measures corrected for plasma volume shift of the respective visit. Of the demographic, life style (including smoking status), and medical variables, triglyceride levels correlated with sICAM- $1_{\mathrm{AUC}}$ at week one $(\mathrm{r}=0.45, \mathrm{p}=0.045, \mathrm{n}=20)$ and screening SBP correlated with SICAM-1 $1_{\mathrm{AUC}}$ at week three $(\mathrm{r}=0.46, \mathrm{p}=0.030, \mathrm{n}=22)$.

\section{Discussion}

We found that sICAM-1 is responsive to acute psychosocial stress in apparently healthy subjects corroborating previous findings (Dugue et al. 1999, Heinz et al. 2003). The lack of a change in sICAM-1 in the non-stress control group supports the notion that the stress-induced change in sICAM-1 was a specific response to the task and, moreover, is in agreement with previous studies suggesting absence of a circadian rhythm in sICAM-1 (Osmančík et al. 2004). Restoration of stress-induced sICAM-1 increase was achieved within 45 min post-stress, compatible with previous recovery of increased sICAM-1 levels after dynamic exercise within one hour (Rehman et al. 1997). Although significant, the relative increases in sICAM-1 from rest to immediately post-stress was only modest $(3.5 \%)$ and therefore comparably lower than the $10-12 \%$ increase in sICAM-1 previously observed in other psychological stress studies (Dugue et al. 1999, Heinz et al. 2003). Moreover, physical stress provoked an even greater sICAM-1 increase of $24 \%$ (Rehman et al. 1997). This difference is perhaps best explained by greater shear forces and subsequently more shedding of ICAM-1 from endothelial cells (Sultan et al. 2004) with exercise compared to psychosocial stress and adrenergic infusions (Rehman et al. 1997), although this needs to be established.

In accordance with previous studies (Schommer et al. 2003), the magnitude of cortisol response to stress significantly decreased from visit one to visit three and the same adaptation effect was observed for SBP response but not for the sICAM-1 stress response. A parsimonious interpretation could be that the magnitude of sICAM-1 increase following repeated acute stress shows no ready habituation but that a possible adaptation could still occur after a series of additional stress repeats.
Theoretically, a lack in habituation of biological stress responses is thought to contribute to various somatic diseases (McEwen 1998). In this case, an equally extensive rise in circulating ICAM-1 e.g. to repetitive daily hassles for decades could contribute to exaggerated vascular inflammatory responses, thereby accelerating atherosclerosis.

We identified several predictors of the total sICAM-1 stress response. However, these results must be interpreted within the context of the particular information that AUC measures used for these analyses can yield. The AUC of a variable provides an easy-tointerpret value of the total integrated activity of a variable over time but no information about the pattern of this change (Pruessner et al. 2003). In terms of determinants of sICAM-1 stress responsiveness, we found that subjects who perceived cognitively more stress in relation to the TSST experienced a greater increase in the integrated sICAM-1 response during stress and recovery. We interpret the lack of a relationship between stress perception and integrated SICAM-1 response at visit three as a consequence of the observed decrease in retrospective stress appraisal between visits. On the retrospective appraisal scale the stressor had reduced novelty and subjects were less eager to perform well at visit three. We have previously shown that more emotional involvement expressed as higher levels of vigorous mood was positively associated with physiological stress responses (von Känel et al. 2005).

We found no significant relationship between integrated stress responses of cortisol and sICAM-1 for both visits. This observation is in line with similar findings from previous stress studies (Heinz et al. 2003, Timmon et al. 2006) but contradicts findings from in vitro studies (Antonelli et al. 2000). Currently, we have little evidence to assume that HPA axis activity significantly affects sICAM-1 levels during both acute psychological and physical stress in vivo. We do not feel that the effect of the HPA axis activity on the SICAM-1 stress response was affected by the cortisol awakening response (CAR) or sleep both of which we did not assess in our subjects. The CAR was previously not associated with subjective sleep quality and sleep duration, and it peaks $30 \mathrm{~min}$ after awakening (Clow et al. 2004), i.e. considerably earlier than the time elapsed between subjects waking up and undergoing the TSST.

In contrast to cortisol reactivity, hemodynamic reactivity, as evidenced by $\mathrm{HR}$ and DBP responses, was significantly related to the integrated SICAM-1 response 
at visit three. HR reflects $\beta_{1}$-adrenergic activity that has been shown to increase sICAM-1 during exercise (Rehman et al. 1997). Moreover, BP increase accelerates blood flow velocity that, in turn, was inversely associated with sICAM-1 levels (Bencze et al. 2006). These mechanisms could help to explain why sICAM-1 change was positively related to HR and inversely related to DBP with stress. In epidemiological studies, sICAM-1 correlated with a range of sociodemographic, life style, and cardiovascular risk factors (Rohde et al. 1999). Our analyses showed that common covariates of sICAM-1 levels did not essentially affect our findings.

Contrary to a previous psychological stress study (Dugue et al. 1999), but compatible with a recent physical exercise study (Timmons et al. 2006), the significant increase in sICAM-1 turned into a significant decrease from rest to immediately post-stress with adjustment of post-stress sICAM-1 levels for plasma volume change. Acute laboratory stress paradigms consistently elicit hemoconcentration (Allen and Patterson 1995) which may evidently affect the time course of different variables of the biological stress response (Bacon et al. 2004, Marsland et al. 1997, Mischler et al. 2005). Plasma volume correction is advised for molecules with a molecular weight of above $69 \mathrm{kDa}$ (Bacon et al. 2004) because these are unable to move passively through endothelial pores with plasma (Berne and Levy 1988). Circulating isoforms of ICAM-1 weight at least $240 \mathrm{kDA}$ and even under denaturating conditions sICAM-1 had a single molecular weight of $80 \mathrm{kDa}$ (Seth et al. 1991). The increase in sICAM-1 to the TSST must therefore be interpreted as a passive phenomenon due to plasma volume contraction rather than an active increase. Admittedly, this reasoning may challenge the aforementioned notion of a shedding process involved in sICAM-1 increase with acute stress, and even more so in the light of a study showing that a 6-min speech did not induce ICAM-1 expression on lymphocytes (Mills and Dimsdale 1996). However, both passive and active processes might be at work because a previous study found a significant increase in sICAM-1 to a psychosocial stressor in spite of adjustment for hemoconcentration (Dugue et al. 1999). That study applied a naturalistic stressor lasting double the time of the TSST in our study, suggesting that the characteristics and duration of the stressor could be responsible for disparities between studies.

We address several limitations of our study. First, our sample was rather small therefore preventing us from extensive statistical controlling of covariates in regression analyses to prevent from overcontrolling (Babyak 2004). Second, the relationship between sICAM-1 stress responsiveness and stress perception on the one hand and hemodynamic reactivity on the other was not significant for both visits. While adaptation in stress perception between visits is a reasonable explanation for this finding, the reason why hemodynamic activity should predict SICAM-1 response at visit three more strongly than at visit one is unclear. Third, the light breakfast served to participants before they were stressed might have affected the absolute values of sICAM-1 though not necessarily its stress response. Fourth, our stress questionnaire bases on welljustified theoretical grounds, but it was self-designed and currently lacks an evaluation of its reliability and validity. Fifth, our findings refer to an apparently healthy and well-educated middle-aged male sample and should not be generalized to women, or populations with cardiovascular diseases and lower socioeconomic status.

In conclusion, we confirmed acute stress responsiveness of sICAM-1 which was to some extent determined by stress appraisal and hemodynamic activity but not by activity of the HPA axis during stress and recovery. The magnitude of the sICAM-1 stress response was not subject to adaptation across stress visits, but was greatly affected by stress-hemoconcentration.

\section{Acknowledgements}

This study was supported by grant 3200-068277 of the Swiss National Science Foundation and by the Swiss Federal Institute of Technology, Zurich.

\section{References}

ALLEN MT, PATTERSON SM: Hemoconcentration and stress: a review of physiological mechanisms and relevance for cardiovascular disease risk. Biol Psychol 41: 1-27, 1995.

ANTONELLI A, BIANCHI M, CRINELLI R, GENTILINI L, MAGNANI M: Modulation of ICAM-1 expression in ECV304 cells by macrophage-released cytokines. Blood Cells Mol Dis 27: 978-991, 2000. 
BABYAK MA: What you see may not be what you get: a brief, nontechnical introduction to overfitting in regressiontype models. Psychosom Med 66: 411-421, 2004.

BACON SL, RING C, LIP GY, CARROLL D: Increases in lipids and immune cells in response to exercise and mental stress in patients with suspected coronary artery disease: effects of adjustment for shifts in plasma volume. Biol Psychol 65: 237-250, 2004.

BENCZE J, KISS RG, TOTH-ZSAMBOKI E, VARGOVA K, KERECSEN G, KORDA A, MOLNAR F, PREDA I: Inverse correlation between coronary blood flow velocity and sICAM-1 level observed in ischemic heart disease patients. Atherosclerosis 188: 142-146, 2006.

BERNE RM, LEVY MN: The Cardiovascular System - The Microcirculation and Lymphatics. In: Physiology. Mosby, St. Louis, Missouri 1988, pp 502-503.

CLOW A, THORN A, EVANS P, HUCKLEBRIDGE F. The awakening cortisol response: methodological issues and significance. Stress 7: 29-37, 2004.

DAVIES MJ, GORDON JL, GEARING AJ, PIGOTT R, WOOLF N, KATZ D, KYRIAKOPOULOS A: The expression of the adhesion molecules ICAM-1, VCAM-1, PECAM, and E-selectin in human atherosclerosis. J Pathol 171: 223-229, 1993.

DILL DB, COSTILL DL: Calculation of percentage changes in volumes of blood, plasma, and red cells in dehydration. J Appl Physiol 37: 247-248, 1974.

DUGUE B, LEPPANEN E, GRASBECK R: Preanalytical factors (biological variation) and the measurement of serum soluble intercellular adhesion molecule-1 in humans: influence of the time of day, food intake, and physical and psychological stress. Clin Chem 45: 1543-1547, 1999.

GAAB J, ROHLEDER N, NATER UM, EHLERT U: Psychological determinants of the cortisol stress response: the role of anticipatory cognitive appraisal. Psychoneuroendocrinology 30: 599-610, 2005.

HAIM M, TANNE D, BOYKO V, RESHEF T, GOLDBOURT U, LEOR J, MEKORI YA, BEHAR S: Soluble intercellular adhesion molecule-1 and long-term risk of acute coronary events in patients with chronic coronary heart disease. Data from the Bezafibrate Infarction Prevention (BIP) Study. J Am Coll Cardiol 39: 1133-1338, 2002.

HEINZ A, HERMANN D, SMOLKA MN, RIEKS M, GRAF KJ, POHLAU D, KUHN W, BAUER M: Effects of acute psychological stress on adhesion molecules, interleukins and sex hormones: implications for coronary heart disease. Psychopharmacology 165: 111-117, 2003.

KAMARCK TW, DEBSKI TT, MANUCK SB: Enhancing the laboratory-to-life generalizability of cardiovascular reactivity using multiple occasions of measurement. Psychophysiology 37: 533-542, 2000.

KIRSCHBAUM C, PIRKE KM, HELLHAMMER DH: The 'Trier Social Stress Test' - tool for investigating psychobiological stress responses in a laboratory setting. Neuropsychobiology 28: 76-81, 1993.

KUROKI K, TAKAHASHI HK, IWAGAKI H, MURAKAMI T, KUINOSE M, HAMANAKA S, MINAMI K, NISHIBORI $\mathrm{M}$, TANAKA $\mathrm{N}$, TANEMOTO $\mathrm{K}$ : $\beta_{2}$-adrenergic receptor stimulation-induced immunosuppressive effects possibly through down-regulation of co-stimulatory molecules, ICAM-1, CD40 and CD14 on monocytes. $J$ Int Med Res 32: 465-483, 2004.

LAZARUS RS, FOLKMAN S: Stress, Appraisal, and Coping. Springer, New York, 2004.

LEEUWENBERG JF, SMEETS EF, NEEFJES JJ, SHAFFER MA, CINEK T, JEUNHOMME TM, AHERN TJ, BUURMAN WA: E-selectin and intercellular adhesion molecule-1 are released by activated human endothelial cells in vitro. Immunology 77: 543-549, 1992.

LEONE M, BOUTIERE-ALBANESE B, VALETTE S, CHAMOIN-JAU L, BARRAU K, ALBANESE J, MARTIN C, DIGNAT-GEORGE F: Cell adhesion molecules as a marker reflecting the reduction of endothelial activation induced by glucocorticoids. Shock 21: 311-314, 2004.

MACCHIONI P, BOIARDI L, MELICONI R, SALVARANI C, GRAZIA UGUCCIONI M, ROSSI F, PULSATELLI L, FACCHINI A: Elevated soluble intercellular adhesion molecule 1 in the serum of patients with polymyalgia rheumatica: influence of steroid treatment. $J$ Rheumatol 21: 1860-1864, 1994.

MARLIN SD, SPRINGER TA: Purified intercellular adhesion molecule-1 (ICAM-1) is a ligand for lymphocyte function-associated antigen 1 (LFA-1). Cell 51: 813-819, 1987. 
MARSLAND AL, HERBERT TB, MULDOON MF, BACHEN EA, PATTERSON S, COHEN S, RABIN B, MANUCK SB: Lymphocyte subset redistribution during acute laboratory stress in young adults: mediating effects of hemoconcentration. Health Psychol 16: 341-348, 1997.

MCEWEN BS: Protective and damaging effects of stress mediators. N Engl J Med 338: 171-179, 1998.

MILLS PJ, DIMSDALE JE: The effects of acute psychologic stress on cellular adhesion molecules. $J$ Psychosom Res 41: 49-53, 1996.

MILLS PJ, FARAG NH, PEREZ C, DIMSDALE JE: Peripheral blood mononuclear cell CD62L and CD11a expression and soluble interstitial cell adhesion molecule-1 levels following infused isoproterenol in hypertension. J Hypertens 20: 311-316, 2002.

MISCHLER K, FISCHER JE, ZGRAGGEN L, KUDIELKA BM, PRECKEL D, VON KÄNEL R: The effect of repeated acute mental stress on habituation and recovery responses in hemoconcentration and blood cells in healthy men. Life Sci 77: 1166-1179, 2005.

MITTLEMAN MA, MACLURE M, TOFLER GH, SHERWOOD JB, GOLDBERG RJ, MULLER JE: Triggering of acute myocardial infarction by heavy physical exertion. Protection against triggering by regular exertion. Determinants of myocardial infarction onset study investigators. N Engl J Med 329: 1677-1683, 1993.

MITTLEMAN MA, MACLURE M, SHERWOOD JB, MULRY RP, TOFLER GH, JACOBS SC, FRIEDMAN R, BENSON H, MULLER JE: Triggering of acute myocardial infarction onset by episodes of anger. Determinants of Myocardial Infarction Onset Study Investigators. Circulation 92: 1720-1725, 1995.

NIEBAUER J, COOKE JP: Cardiovascular effects of exercise: role of endothelial shear stress. J Am Coll Cardiol 28: 1652-1660, 1996.

O'MALLEY T, LUDLAM CA, RIEMERSMA RA, FOX KA: Early increase in levels of soluble inter-cellular adhesion molecule-1 (sICAM-1); potential risk factor for the acute coronary syndromes. Eur Heart $J$ 22: 1226-1234, 2001.

OSMANČÍK P, KVASNIČKA J, WIDIMSKÝ P, TARNOK A: Diurnal variation of soluble E- and P-selectin, and intercellular adhesion molecule-1 in patients with and without coronary artery disease. Cardiology 102: 194199, 2004.

PARK CL: Stress-related growth and thriving through coping: The roles of personality and cognitive processes. $J$ Soc Issues 54: 267-277, 1998.

PRADHAN AD, RIFAI N, RIDKER PM: Soluble intercellular adhesion molecule-1, soluble vascular adhesion molecule-1, and the development of symptomatic peripheral arterial disease in men. Circulation 106: 820-825, 2002.

PRICE DT, LOSCALZO J: Cellular adhesion molecules and atherogenesis. Am J Med 107: 85-97, 1999.

PRUESSNER JC, KIRSCHBAUM C, MEINLSCHMID G, HELLHAMMER DH: Two formulas for computation of the area under the curve represent measures of total hormone concentration versus time-dependent change. Psychoneuroendocrinology 28: 916-931, 2003.

REHMAN J, MILLS PJ, CARTER SM, CHOU J, THOMAS J, MAISEL AS: Dynamic exercise leads to an increase in circulating ICAM-1: further evidence for adrenergic modulation of cell adhesion. Brain Behav Immun 11: 343351, 1997.

RIDKER PM, HENNEKENS CH, ROITMAN-JOHNSON B, STAMPFER MJ, ALLEN J: Plasma concentration of soluble intercellular adhesion molecule 1 and risks of future myocardial infarction in apparently healthy men. Lancet 351: 88-92, 1998.

ROHDE LE, LEE RT, RIVERO J, JAMACOCHIAN M, ARROYO LH, BRIGGS W, RIFAI N, LIBBY P, CREAGER MA, RIDKER PM: Circulating cell adhesion molecules are correlated with ultrasound-based assessment of carotid atherosclerosis. Arterioscler Thromb Vasc Biol 18: 1765-1770, 1998.

ROHDE LE, HENNEKENS CH, RIDKER PM: Cross-sectional study of soluble intercellular adhesion molecule-1 and cardiovascular risk factors in apparently healthy men. Arterioscler Thromb Vasc Biol 19: 1595-1599, 1999.

SCHOMMER NC, HELLHAMMER DH, KIRSCHBAUM C: Dissociation between reactivity of the hypothalamuspituitary-adrenal axis and the sympathetic-adrenal-medullary system to repeated psychosocial stress. Psychosom Med 65: 450-460, 2003. 
SETH R, RAYMOND FD, MAKGOBA MW: Circulating ICAM-1 isoforms: diagnostic prospects for inflammatory and immune disorders. Lancet 338: 83-84, 1991.

SULTAN S, GOSLING M, NAGASE H, POWELL JT: Shear stress-induced shedding of soluble intercellular adhesion molecule-1 from saphenous vein endothelium. FEBS Lett 564: 161-165, 2004.

TIMMONS BW, HAMADEH BW, TARNOPOLSKY MA: No effect of short-term 17 $\beta$-estradiol supplementation in healthy men on systemic inflammatory responses to exercise. Am J Physiol 291: R285-R290, 2006.

VON KÄNEL R, PRECKEL D, ZGRAGGEN L, MISCHLER K, KUDIELKA BM, HAEBERLI A, FISCHER JE: The effect of natural habituation on coagulation responses to acute mental stress and recovery in men. Thromb Haemost 92: 1327-1335, 2004.

VON KÄNEL R, KUDIELKA BM, PRECKEL D, HANEBUTH D, HERRMANN-LINGEN C, FREY K, FISCHER JE: Opposite effect of negative and positive affect on stress procoagulant reactivity. Physiol Behav 86: 61-68, 2005.

VON KÄNEL R, KUDIELKA BM, PRECKEL D, HANEBUTH D, FISCHER JE: Delayed response and lack of habituation in plasma interleukin-6 to acute mental stress in men. Brain Behav Immun 20: 40-48, 2006.

WANG JH, DEVALIA JL, SAPSFORD RJ, DEVIES RJ: Effect of corticosteroids on release of RANTES and sICAM1 from cultured human bronchial epithelial cells, induced by TNF-alpha. Eur Respir J 10: 834-840, 1997.

WERTHEIMER SJ, MYERS CL, WALLACE RW, PARKS TP: Intercellular adhesion molecule-1 gene expression in human endothelial cells. Differential regulation by tumor necrosis factor-alpha and phorbol myristate acetate. J Biol Chem 267: 12030-12035, 1992.

WESTERMAN J, DEMIR A, HERBST V: Determination of cortisol in saliva and serum by a luminescence-enhanced enzyme immunoassay. Clin Lab 50: 11-24, 2004.

WILSON PW, d'AGOSTINO RB, LEVY D, BELANGER AM, SILBERSHATZ H, KANNEL WB: Prediction of coronary heart disease using risk factor categories. Circulation 97: 1837-1847, 1998.

WITKOWSKA AM: Soluble ICAM-1: a marker of vascular inflammation and lifestyle. Cytokine 31: 127-134, 2005.

\section{Corresponding author}

Roland von Känel, Department of General Internal Medicine, University Hospital/Inselspital, CH-3010 Bern, Switzerland. Fax +41 3138211 84. E-mail: roland.vonkaenel@insel.ch 